\title{
Kulturelle Einflüsse auf die europäische betriebliche Mitbestimmung
}

Die Etablierung von Eurobetriebsräten (EBR) ist ein Schritt zur Internationalisierung der Vertretung von Arbeitnehmerinteressen. Doch was passiert, wenn sich betriebliche Arbeitnehmervertreter aus unterschiedlichen Ländern treffen, um sich zu verständigen und gemeinsame Strategien zu entwickeln? Karl Marx hat einmal geschrieben, der Arbeiter kenne kein Vaterland, sondern nur proletarische Interessen. Aber so einfach ist es nicht. Strategien, die sich national entwickelt und bewährt haben, treffen auf der europäischen Ebene auf Strategien aus anderen Ländern. Nicht nur Interessen, sondern auch kulturelle Muster der Interessenvertretung beeinflussen das Gelingen oder Misslingen europäischer Arbeitnehmervertretung. ${ }^{1}$

\section{Einleitung}

Die Transnationalisierung großer Unternehmen stellt die betriebliche Vertretung von Arbeitnehmerinteressen vor neue Herausforderungen. Einerseits werden Betriebsräte $^{2}$ in zunehmendem Maße mit der internationalen Orientierung ihres Managements konfrontiert, das mithilfe glaubhafter Androhungen von Produktionsverlagerungen oder gar der Schließung ganzer Standorte ein, wie Hermann Kotthoff meint, "gnadenloses Kostensenkungsdiktat" (Kotthoff 1998) innerhalb transnationaler Unternehmensstrukturen betreibt. Andererseits bietet die 1996 erfolgte Umsetzung der Europäischen Richtlinie zur Einrichtung von Eurobetriebsräten (EBR) in deutsches Recht die Möglichkeit, ein länderübergreifendes Betriebsratsgremium zu gründen, das dann allerdings auch mit Leben gefüllt werden muss. $^{3}$

Wenn die einzelbetriebliche Wirksamkeit der Interessenvertretung in Deutschland an Grenzen stößt, weil die Reichweiten des Betriebsratshandelns und des Managementhandelns auseinanderklaffen, liegt die Vermutung nahe, dass Betriebsräte (BR) neu entstehende internationale Gremien nutzen werden, um ihren Einfluss auf das Management wieder stärker zur Geltung zu bringen. Eurobetriebsräte werden damit zu Foren, in denen nicht nur oder in erster Linie eine europäische Identität der Arbeitnehmer vermittelt wird, sondern mit deren Hilfe Handlungsmöglichkeiten im nationalen Raum gestärkt werden sollen.
Ob dies gelingt, hängt von dem Vermögen ab, die neue, ,transnationale “ Handlungsarena in der Kommunikation mit den Belegschaftsvertretern anderer Länder fruchtbar zu machen. Im Folgenden wollen wir auf der Grundlage der im Rahmen eines empirischen Forschungsprojektes gewonnenen Erkenntnisse ${ }^{4}$ zeigen, dass deutsche Betriebsräte den Eurobetriebsrat dazu nutzen, verloren gegangene Interessenvertretungswirksamkeit zu restaurieren. Sie probieren dies durch eine Ausdehnung ihres Vertretungsmandats auf ausländische Standorte, d.h. sie versuchen, sich als Sprecher für andere Standorte gegenüber dem zentralen Management zu etablieren und das national bewährte Modell des Stellvertretungshandelns auf die europäische Ebene zu übertragen. Die zu beobachtenden Probleme in der Zusammenarbeit in den EBR können dabei nicht alleine auf Standortkonkurrenzen zurückgeführt werden, sondern beruhen auch auf „Fallstricken“ in der interkulturellen Kommunikation zwischen Interessenvertretern, die über unterschiedliche Selbstverständnisse und Erfahrungen betrieblicher Mitbestimmung verfügen. Dies zeigen wir anhand des Verhältnisses deutscher EBR-Mitglieder zu ihren mittel- und osteuropäischen Kollegen.

Bevor wir unsere Argumentation am empirischen Material entwickeln (Abschnitt 4), zeichnen wir zunächst die bisherige Diskussion um Probleme der Zusammenarbeit von Arbeitnehmerver-

\footnotetext{
1 Der Artikel basiert auf einem Beitrag für das Plenum der Sektion Arbeits- und Industriesoziologie am 15.10.2010 im Rahmen des Kongresses der Deutsche(n) Gesellschaft für Soziologie (DGS) "Transnationale Vergesellschaftungen."
}

2 Im Folgenden verwenden wir der Kürze halber nur die männliche Form, das andere Geschlecht ist natürlich mitgemeint.

3 Zur Entstehungsgeschichte der Richtlinie siehe Lecher et al. (1998) und Kotthoff (2006). In etwa $37 \%$ der Unternehmen, in denen ein EBR möglich wäre, wurde ein EBR gegründet (vgl. Hauser-Ditz et al. 2010). Zu den methodischen Problemen der Erfassung und den Gründen für die niedrige Quote bei deutschen Unternehmen siehe Lücking et al. (2008) und Whittall et al. (2008).

4 Das Projekt wurde unter dem Titel „Chancen kulturübergreifender Solidarität in global agierenden Unternehmen: Kommunikation, Macht und Interessen in der transnationalen Arbeitnehmerkooperation" von 2006 bis 2009 von der Hans-Böckler-Stiftung gefördert (Projektnr S-2006-866-2 F) und an der Universität Erlangen-Nürnberg unter Leitung von Gert Schmid und Ilja Srubar durchgeführt. Eine Publikation des Projektabschlussberichtes (vgl. dazu Klemm et al. 2011) ist in Vorbereitung.

Matthias Klemm, Dr., wissenschaftlicher Mitarbeiter am Institut für Soziologie, Universität Erlangen-Nürnberg und am Institut für Soziologie, Universität Münster. Arbeitsschwerpunkte: Kultur- und Wissenssoziologie, Arbeits- und Organisationsforschung.

e-mail: msklemm@phil.uni-erlangen.de Clemens Kraetsch, M.A., wissenschaftlicher Mitarbeiter am Institut für Soziologie, Universität Erlangen-Nürnberg und am Institut für Soziologie, Universität Münster. Arbeitsschwerpunkte: Arbeit, Organisation, Industrielle Beziehungen. e-mail: clemens.kraetsch@soziol.phil.unierlangen.de Jan Weyand, Dr., wissenschaftlicher Mitarbeiter am Institut für Soziologie, Universität Erlangen-Nürnberg. Arbeitsschwerpunkte: Soziologische Theorie, Kultursoziologie, Antisemitismusforschung. e-mail: jnweyand@phil.uni-erlangen.de. 
tretern in einem interkulturellen Gremium nach (Abschnitt 2) und stellen die theoretischen Ausgangsüberlegungen sowie das zugrunde liegende Forschungsprojekt vor (Abschnitt 3). Wir schließen mit einem Fazit (Abschnitt 5).

\section{Kulturelle Einflüsse als Analysedimension für EBR-Handeln - Forschungsstand}

Seit der Einführung der EBR wurde eine Reihe von Studien zur Zusammenarbeit in Eurobetriebsräten und der Wirksamkeit der Gremien gegenüber dem Management durchgeführt (als Überblick zur Forschung siehe u.a. Müller/Hoffmann 2001 und Hauser-Ditz et al. 2010). Sieht man von Studien zur Vorgeschichte der EURichtlinie ab, zeichnet sich das Gros der Literatur durch auf Fallstudien beruhenden Einschätzungen und Typologien zur Wirksamkeit und Arbeitsfähigkeit von EBR aus (z.B. Lecher et al. 1998, 2001; Eberwein et al. 2000; Kotthoff 2006; Hauser-Ditz et al. 2010). Hierbei werden neben eher personellen Faktoren strukturelle Faktoren (wie Unternehmensstruktur, Branche etc.) identifiziert, die die Gestaltung der internationalen Arbeitnehmervertretung beeinflussen. Die zu beobachtenden Konflikte innerhalb der EBR werden hauptsächlich mit Interessengegensätzen aufgrund von Standortkonkurrenzen erklärt. Auch auf Probleme zwischen deutschen und nichtdeutschen EBR-Mitgliedern infolge länderspezifisch unterschiedlicher Ansichten zum Umgang mit dem Management wird verwiesen (vgl. Kotthoff 2006). Die Analyse „kultureller“ Einflussfaktoren bleibt meist bei der Feststellung unterschiedlicher Sichtweisen, Formen der Diskussionskultur oder Denkweisen stehen (Ausnahme: Stirling/Tully 2004). Aktuell haben HauserDitz et al. (2010) für die Analyse ihrer Fallstudien zur Wirksamkeit unterschiedlicher Typen von Eurobetriebsräten ein komplexes Kategorienset entwickelt, in dem institutionelle und kulturelle Länderkontexte zu einem Einflussfaktor (von vier wichtigen) zusammengefasst sind. Diese werden über die jeweilige nationale Ausgestaltung der Industriellen Beziehungen und der Unternehmenskulturen konzeptualisiert. Gemeinsam ist den genannten Untersu- chungen, dass kulturelle und sprachliche Verständigungshindernisse anerkannt werden, worin diese jedoch genau bestehen, bleibt weitgehend unbestimmt. Eine Sonderstellung nehmen in dieser Hinsicht die Untersuchungen von Kotthoff ein: Hier werden kulturelle Einflussfaktoren zwar aufgezeigt, aber letztlich - aufgrund eingeschliffener Kooperationsroutinen - als überwunden betrachtet. So hätten etwa deutsche EBR-Mitglieder „schnell gelernt, zurückhaltender, kleinlauter, vor allem geduldiger zu sein. Sie haben gemerkt, dass sie Fremde unter Fremden sind, und sie haben gelernt, mit ihrer Vorreiterrolle, die sie häufig nach wie vor faktisch einnehmen, anders umzugehen " (Kotthoff 2006, S. 120).

Ein ähnliches Bild ergibt sich bei Studien, die das Verhältnis zwischen westeuropäischen bzw. deutschen Eurobetriebsräten und ihren mittel- und osteuropäischen Kollegen thematisieren. Kotthoff (2005) sieht in seinen Fallstudien die West-OstKonkurrenz als das ,prägende Dauerthema im Umgang mit den Delegierten aus den MOE-Ländern" (Kotthoff 2005, S. 30). Eine Zusammenarbeit sei nur durch ,eine Reihe von mentalen und sozialen Konstrukten “ möglich, „die das Verbindende vergrößern und das Trennende verkleinern" (Kotthoff 2005, S. 48). Voss (2006) verbindet seine Studie u.a. mit der Frage, wie nach der EU-Erweiterung 2004 die Eurobetriebsräte mit der erhöhten sozio-ökonomischen und kulturellen Vielfalt/Verschiedenartigkeit umgehen können. Aktuell ist Dehnen (2010) anhand des Fallbeispiels General Motors auf die Einbindung polnischer betrieblicher Arbeitnehmervertreter in die EBR-Arbeit eingegangen. Sie kommt zu dem Schluss, dass die „Osterweiterung" der EBR neue Konfliktlinien habe entstehen lassen. Der wichtigste Konflikt sei hierbei der um Produktionsvolumina, der sich aber lösen lasse, wenn nationale Unterschiede benannt würden und in die Formulierung der EBR-Erklärungen und Strategien einflössen. Neben dem HauptKonflikt um Produktionsvolumina wird nur kurz und sehr allgemein auf andere Gründe für die Probleme der Zusammenarbeit verwiesen: Die geringen Kenntnisse über die anderen, Verständigungsschwierigkeiten und Vorurteile (Dehnen 2010, S. 167). Hürtgen (2008) argumentiert, dass durch die transnationale Wirkung kapitalistischer Verwertungs- und Konkurrenzlogiken längst ein ebenso transnationales wie defensives gemeinsames Verständnis des Interessenvertretungshandelns in Europa entstanden sei, dass auf die Verteidigung "guter Arbeit" abziele. Durch diese strukturell erzwungene Vereinheitlichung der Sichtweisen würden nationalkulturelle und institutionelle Unterschiede in der Interessenvertretung in den Hintergrund treten. Eine „kulturalistische“ Argumentation gehe insofern an den ökonomischen und politischen Handlungszwängen, denen die Akteure unterworfen sind, vorbei.

Im Folgenden wird zu zeigen sein, dass bei einer präzisen Fassung der Problematiken interkultureller Kommunikation der ansonsten berechtigte Vorwurf einer „kulturalistischen" Sichtweise widerlegt werden kann und Erkenntnisse aus der Kulturforschung helfen, die Probleme und Friktionen von Kooperationen im Rahmen des EBR besser zu verstehen.

\section{3 \\ Vertretungshandeln deut- scher BR im europäischem Rahmen - Theorie, Metho- de, empirische Grundlage}

\subsection{THEORETISCHE ÜBERLEGUNGEN}

Die Berücksichtigung kultureller Einflüsse auf die Kooperation von Eurobetriebsräten ergänzt das völlig unstrittige Faktum, dass Auseinandersetzungen zwischen Kapital und Arbeit, aber auch zwischen Arbeitnehmervertretern unterschiedlicher Standorte und Länder, immer auch Auseinandersetzungen um divergierende Interessen sind. Arbeitnehmervertreter sind Interessenvertreter, und die Erweiterung der Chancen, die eigenen Interessen bzw. die Interessen der eigenen Belegschaften - im Zweifelsfalle auch gegen die Interessen anderer Belegschaften - durchsetzen zu können, gehört zum „Kerngeschäft" von Betriebsräten und Gewerkschaftern. Die Rolle von „Kultur“ muss daher innerhalb von Macht- und Interessenbeziehungen gesucht werden, nicht jenseits oder alternativ zu diesen.

Zur Durchsetzung von Interessen bedarf es spezifischer Mittel, sei es der Drohung, der Verhandlung oder der Konzession. Diese Mittel werden in der Regel nicht erst im Ernstfall erfunden, sondern liegen gewissermaßen als „Werkzeugkasten“ bereits vor. Die einzelnen „tools“, etwa Streiks, Betriebsversammlungen, Reden, 
Verhandlungstaktiken etc., sind auch kulturelle Praktiken angemessenen Handelns, Deutens und Redens (Swidler 1986). Die europaweit unterschiedlichen, national "gewachsenen“ Praktiken der Interessenvertretung haben diesbezüglich eines gemeinsam: In den jeweiligen Ländern gelten sie für die meisten Akteure als die nahe liegenden, selbstverständlichen und zumeist auch angemessenen Formen der Ordnung von Interessengegensätzen und des Handelns in Konfliktlagen. Diese sozial gebilligten „Werkzeugkästen“ bezeichnen wir als Mitbestimmungskulturen, die im Rahmen von Interessens- und Machtprozessen für Akteure zur Verfügung stehen. Und um Ähnlichkeiten und Unterschiede dieser Werkzeugkästen geht es uns im Folgenden. Allgemein lässt sich ein solcher kultureller Werkzeugkasten nach drei Dimensionen gliedern:

(1) Formen von Selbst- und Fremdbildern (Einschätzungen, Bewertungen und Identitäten): Hierunter fallen kollektive Formen der Weltwahrnehmung, die historisch und kulturell über die Etablierung von Sozialräumen in Abgrenzung zu anderen Kollektiven entstehen: etwa unterschiedliche Geschmäcker, Formen der Distinktion (Bourdieu) und Praktiken der Selbstdarstellung. So zeigen etwa sozialstrukturell orientierte Untersuchungen mithilfe des Milieu-Konzeptes, dass unterschiedliche soziale Lagen mit unterschiedlichen Geschmacksvorstellungen einhergehen.

(2) Formen des Handelns (Mittel des Durchsetzens von Interessen): Milieus und Kulturen sind sichtbar in unterschiedlichen Handlungsstilen. Beispiele wären etwa die sichtbar unterschiedlichen Formen katholischer und evangelischer Gottesdienste, der Intensität alltäglicher Religiosität etc.

(3) Formen des Kommunizierens (Mittel des Überzeugens, Verhandlung, Aushandlung): Hierbei sind zu nennen die Ausdrucksmittel, allen voran die Sprache und ihr Repertoire zur Bezeichnung richtigen und falschen Verhaltens, die Kodes der Herstellung von Konsens, die Anzeige von Zugehörigkeit und die Definitionsmacht dessen, was legitime und illegitime Handlungsmittel und -ziele sind.

Mithilfe dieses hier sehr knapp skizzierten Kulturverständnisses lassen sich zwei „Einfallstore“ von Kultur in die Koopera- tion von europäischen Arbeitnehmervertretern identifizieren: einerseits hinsichtlich der Fragen der Verständigung und Interpretation der jeweils anderen, andererseits hinsichtlich der Definitionshoheit über den gemeinsamen Handlungs- und Kommunikationsraum Europa bzw. die infrage stehenden transnationalen Unternehmen. Im ersten Fall ist offen, wie national erprobte Strategien und Handlungsweisen von den "anderen“ wahrgenommen, rezipiert und kommentiert werden. Im zweiten Fall bleibt unklar, inwieweit verschiedene Seiten die Möglichkeit erhalten, ihre Sicht auf gemeinsame Handlungsprobleme zum Ausdruck zu bringen. Geht es im ersten Falle um interkulturelle Kommunikation im engeren Sinne, so geht es im zweiten Fall um die Definitionsmacht im mehr oder minder gemeinsamen Handlungsraum (um „membership space“, vgl. Srubar 1998).

In den folgenden Ausführungen werden wir zeigen, dass beide Dimensionen den Versuch deutscher Betriebsräte beeinflussen, durch eine „Inanspruchnahme“ des EBR ihre national erodierte Interessenvertretungswirksamkeit zurückzugewinnen und damit das Managementhandeln „einzuhegen“.

\subsection{EMPIRISCHE GRUNDLAGE UND METHODEN}

Im Rahmen der bereits erwähnten empirischen Studie „Chancen kulturübergreifender Solidarität in global agierenden Unternehmen: Kommunikation, Macht und Interessen in der transnationalen Arbeitnehmerkooperation" (vgl. Fußnote 4) haben wir in drei Unternehmen der Automobil- und der Automobilzulieferindustrie untersucht, wie Betriebsräte bzw. betriebliche Arbeitnehmervertreter von unterschiedlichen Unternehmensstandorten und aus verschiedenen europäischen Ländern im Rahmen des EBR zusammenarbeiten (vgl. auch Klemm/Weyand 2009). Die Untersuchung kultureller Aspekte der Zusammenarbeit legte ein qualitatives Untersuchungsdesign nahe. Als Untersuchungsinstrumente kamen leitfadengestützte Interviews, teilnehmende Beobachtungen von internationalen Treffen und Dokumentenanalysen zum Einsatz. Es wurden u.a. 44 Experten- sowie leitfadengestützte Interviews mit Angehörigen der betrieblichen Interessenvertretung und mit Gewerkschaftsvertretern geführt, die als EBR-Mitglieder über internationale Kontakte oder durch ihre Stellung in der betrieblichen Interessenvertretung in die europäische Interessenvertretungsarbeit involviert waren. Die betriebsbezogenen Erhebungen fanden an 14 Standorten in fünf Ländern (sechs in Deutschland, einer in Polen, zwei in Spanien, zwei in Tschechien, zwei in Ungarn) statt. Das Übergewicht der deutschen Standorte ist Folge der Unternehmensstrukturen. Die Interviews dienten u.a. der Erhebung von Selbst- und Fremdbildern sowie der Bestimmung von Solidaritätsverständnissen. Alle Interviews wurden in der Muttersprache der Befragten geführt, transkribiert und übersetzt. ${ }^{5}$ Darstellungs-, Kommunikations- und Verhandlungsstile wurden durch die teilnehmende Beobachtung von insgesamt vier größeren internationalen Treffen (drei EBR-Treffen und ein Netzwerktreffen unterhalb der EBR-Ebene) sowie im Zuge von mit Betriebsräten vorgenommenen Betriebsbesuchen und kleineren Treffen erhoben. An allen Treffen nahmen mindestens zwei Vertreter des Forschungsteams teil. $\mathrm{Zu}$ jedem Treffen wurden ausführliche Beobachtungsprotokolle angefertigt.

Sowohl die Erhebung als auch die Auswertung orientierte sich an Annahmen der "grounded theory“: Vorannahmen über Handlungsmotive, Interessen und Strategien in der internationalen Arbeitnehmerinteressenvertretung sollten so weit wie möglich expliziert werden und nur kontrolliert in den Forschungs- und Auswertungsprozess einfließen. Zunächst wurden die erhobenen Daten hermeneutisch, konkreter: fallrekonstruktiv ausgewertet. Im Verlauf der weiteren Auswertung wurden verstärkt typisierende Verfahren eingesetzt, um der Fülle des Materials gerecht zu werden.

Zwei Fragen standen im Mittelpunkt der Auswertung: Mit welchen Selbst- und Fremdbildern gehen Arbeitnehmervertreter aus den verschiedenen Ländern an die Zusammenarbeit heran? Inwieweit gibt es übernational geteilte, gemeinsame Konzeptionen von Solidarität? Von

5 Die Erhebung, Übersetzung und Interpretation von Interviews in verschiedenen Sprachen und aus unterschiedlichen kulturellen Kontexten ist mit verschiedenen Herausforderungen verbunden, auf die wir an dieser Stelle nicht im Einzelnen eingehen können. Eine ausführliche Erläuterung findet sich in Klemm et al. (2011). 
besonderem Interesse war dabei das Verhältnis zwischen den Arbeitnehmervertretern deutscher Standorte auf der einen und von Standorten in Mittelosteuropa (MOE) auf der anderen Seite. Gerade diese Konstellation scheint gegenüber anderen Konstellationen aus der Sicht deutscher Betriebsräte besonders problematisch: Die (angeblichen) Kostenvorteile einer Produktion an den MOE-Standorten werden von den Unternehmensleitungen regelmäBig als Druckmittel für Kosteneinsparungen in Anschlag gebracht, die von den Belegschaften deutscher Standorte erbracht werden sollen.

\subsection{GRENZEN DER GENERA- LISIERUNG}

Die Grenzen der Verallgemeinerbarkeit der folgenden Befunde werden durch drei Charakteristika der untersuchten Fälle bestimmt:

(1) Alle Unternehmen gehören zur Automobil- und Automobilzulieferindustrie, ihr Stammsitz ist in Deutschland und sie haben nach 1990 in Mittelosteuropa Produktionsanlagen aufgebaut, die in der Mehrzahl der Fälle inzwischen in jeder Hinsicht auf dem technischen Stand der etablierten Standorte in Deutschland sind. Hinsichtlich der Beschäftigtenzahl bildet Deutschland den Schwerpunkt und somit wird auch der EBR von (west-)deutscher Seite dominiert.

(2) Die international aktiven Arbeitnehmervertreter deutscher Standorte nehmen in der Regel auch wichtige Positionen in der nationalen betrieblichen Mitbestimmung ein. Sie sind ausnahmslos lange in der Interessenvertretung aktiv und kennen eine Vielzahl von Werksleitern in den Werken in MOE persönlich. Sie haben fast alle einen direkten Zugang zum zentralen Management, deren wichtigste Vertreter ihnen persönlich bekannt sind.

(3) Alle Unternehmen verfügen über einen EBR, der inzwischen auf eine langjährige Erfahrung zurückblicken kann.

Sicherlich sind in anderen EBR-Konstellationen (z.B. nicht-deutsche Unternehmenszentrale, andere Branchen, andere Länderverteilung) andere Vorgehensweisen von deutscher Seite zu erwarten. Gleichwohl vermuten wir, dass die kulturellen Pro- blemlagen, mit denen Eurobetriebsräte konfrontiert sind, strukturell vergleichbar sein dürften. $\mathrm{Ob}$ und inwieweit diese Vermutung zutrifft, müssen zukünftige differenzierte vergleichende Forschungen zur internationalen Kooperation betrieblicher Arbeitnehmervertreter zeigen.

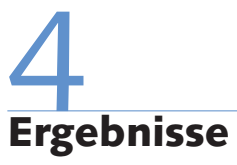

\subsection{ANLASS FÜR DAS DEUTSCHE INTERNATIONALE VERTRETUNGS- HANDELN}

Für deutsche Betriebsräte ist, wie eingangs vermutet, die Zunahme der unternehmensinternen Konkurrenz der Standorte ein herausgehobener Anlass für das Engagement auf der europäischen Ebene betrieblicher Interessenvertretung. Ihnen geht es auf längere Sicht darum, diese Konkurrenz und deren negative Folgen insbesondere für die deutschen Standorte einzudämmen:

„(...) eigentlich (Hervorh. d. V.) sitzen wir alle im selben Boot, also die Interessenvertretung und wir müssen gucken, dass wir bei Expansion das gleichmäßig verteilen, nämlich nicht so unter dem Motto: Wenn es nach oben geht, dann geht es in die Regionen, wo die Löhne und Arbeitsbedingungen nicht so gut sind, (...) und wenn es dann runtergeht, dann fährt man das an den sogenannten teuren Standorten runter, ne..." (deutsches EBR-Mitglied, Fall 1).

Diese, in der deutschsprachigen EBRLiteratur durchgängig hervorgehobene und für mehr oder minder selbstverständlich gehaltene Problemdefinition stellt ein kulturelles Deutungsmuster par excellence dar: Einerseits wird die grundlegende Einsicht der Gemeinsamkeit der Lage von Arbeitnehmern aufgerufen. Andererseits aber bricht sich dies mit einer spezifischen historischen Erfahrung ungleicher Behandlung. Denn bereits wiederholt wurde seitens des Managements versucht, die Arbeits- und Einkommensbedingungen der in der Ausgangssituation bessergestellten deutschen Arbeitnehmer abzusenken. Diese Situationsdefinition wird als gemeinsame Grundlage des europäischen Betriebsratshandelns und somit auch als Grundlage der internationalen Verständigung zwischen Arbeitnehmervertretern vorausgesetzt.
Bis in die Mitte der 1990er Jahre hinein (und teils länger) waren jedoch weder die organisationsseitigen Rahmenbedingungen noch die Deutungsmuster in der Bundesrepublik derart stark auf unternehmensinterne Konkurrenzsituationen fokussiert. In unseren Fällen wurden in den frühen 1990er Jahren die mittelosteuropäischen Arbeitnehmervertreter beim Aufbau der Vertretungsarbeit unterstützt. Diese Unterstützung wird in der Retrospektive als eine Art „Fernsolidarität“ und Aufbauhilfe verstanden. Die Standorte Mittelosteuropas waren auf der „mentalen Landkarte" weit entfernt: Sie wurden nicht oder kaum als Bedrohung betrachtet. Der „Aufbau Ost" wurde im Westen abgesegnet und begrüßt. Erst im Zuge des Ausbaus von Produktionskapazitäten bei weiterhin niedrigen Löhnen wurden die mittelosteuropäischen Standorte zunehmend als Bedrohung für die eigenen Belegschaften wahrgenommen. Die Markteintrittstrategien der Hersteller rücken in den Verdacht der mehr oder minder systematischen Schaffung von Überkapazitäten, die sich in den Händen des Managements als gefährliche Waffe des Standortwettbewerbs darstellen. So sinkt die Fähigkeit der deutschen Arbeitnehmervertreter, Interessen auf lokaler und nationaler Ebene durchzusetzen. Der Kontakt zu und die Kooperation mit Standorten aus Mittel- und Osteuropa bekommt eine veränderte Bedeutung: Sie werden unmittelbar wichtig.

\subsection{STRATEGIEN DES VERTRETUNGS- HANDELNS AUF EUROPÄISCHER EBENE}

Die deutschen Betriebsräte setzen vor diesem Hintergrund verstärkt auf eine Einbindung mittelosteuropäischer Standorte in ihre Vertretungsstrategien. Dabei fällt auf, dass weniger der Weg einer gemeinsamen Verständigung über gemeinsame Ziele angestrebt wird, sondern eine Ausdehnung des eigenen Vertretungsmandats auf die mittel- und osteuropäischen Standorte. Einerseits werden über den Eurobetriebsrat verstärkt Standorte in Mittelosteuropa kontaktiert und besucht. Andererseits werfen die deutschen Vertreter ihre „Hausmacht“ (also ihre nationale Stellung im Verhältnis zur Konzernzentrale) in die Waagschale, um Veränderungen an Auslandsstandorten zu erreichen, deren Durchsetzung vor Ort nicht möglich oder nicht angestrebt war. Dies geschieht z.B. durch 
- Intervention beim zentralen Management, damit dieses das lokale Standortmanagement maßregelt (z.B. bei der Versorgung von Belegschaftsvertretern mit Computern) oder

- direkte Intervention beim Lokalmanagement des Auslandsstandorts (etwa bei der Bereitstellung von Waschgelegenheiten) sowie

- den Versuch, europaweit Standards des Arbeitsschutzes oder des Produktwettbewerbs zu etablieren.

Tatsächlich sind dies drei unterscheidbare Strategien der Interessenvertretung durch den EBR, wie sie auch aus anderen Untersuchungen bekannt sind (z.B. bei Kotthoff 2006, S. 64ff.). In der Forschungsliteratur liegen sie häufig der Typenbildung unterschiedlicher Wirksamkeitsgrade von EBR zugrunde (Lecher et al. 2001, S. 200ff.) oder zeigen unterschiedliche Intensitätsgrade der erreichten internationalen Kooperation und der Vertrautheit der Akteure miteinander an (Kotthoff 2006). Die Dominanz der jeweiligen Strategie im Handeln eines EBR kann zum Teil über die Einbettung des EBR in die Logik der Unternehmensorganisation - eher transnational, eher global, eher multinational - erklärt werden (vgl. Hauser-Ditz et al. 2010). Auch in den von uns untersuchten Fällen werden fallspezifisch entweder stärker Strategien der europaweiten Durchsetzung von Standards oder Interventionen beim zentralen Management oder Interventionen beim Lokalmanagement praktiziert. Für die hier verfolgte Fragestellung ist aber entscheidend, dass die deutschen Betriebsräte im internationalen Rahmen ungeachtet der fallspezifischen Unterschiede allesamt aktiv darum bemüht sind, sich als Vertretung der Interessen aller oder vieler Standorte gegenüber dem Management zu etablieren.

Natürlich dienen diese Maßnahmen auch dazu, illegitimen „Standortvorteilen“, die durch das Unterlaufen von arbeitsrechtlichen Mindeststandards erzielt werden, im Ausland zu begegnen. Sie liegen soweit immer auch im „wohlverstandenen Eigeninteresse" der westdeutschen Arbeitnehmervertreter. Es muss jedoch gesehen werden, dass viele dieser Aktionen in keinem direkten Bezug zum Kostenwettbewerb der Standorte stehen (etwa die europaweite Durchsetzung der Entlohnung von Mitarbeitern für Verbesserungsvorschläge durch deutsche Betriebsräte), sie aber einerseits durchaus als Beitrag zur europäischen Gleichstellung der Arbeitnehmer und andererseits als Vorleistung betrachtet werden, um Arbeitnehmervertreter in Mittel- und Osteuropa für sich zu gewinnen. Das Vorgehen, insbesondere der deutschen Vertreter im EBR, besteht dabei darin, qua Eurobetriebsrat einen europaweiten Vertretungsanspruch hinsichtlich der genannten Forderungen gegenüber dem Management geltend zu machen: Man agiert für die oder an Stelle der lokalen Belegschaftsvertreter.

\subsection{DAS DEUTSCHE VERTRETUNGS- HANDELN IM INTERKULTURELLEN KONTEXT}

Der Anspruch, für alle Arbeitnehmerinnen und Arbeitnehmer eines Unternehmens das Vertretungsmandat innezuhaben, setzt die Bereitschaft auf Seiten der Kollegen aus den Standorten in Mittel- und Osteuropa voraus, sich von den deutschen Vertretern repräsentieren zu lassen und deren Vertretungsanspruch zu unterstützen. In unseren Fallbeispielen zeigt sich jedoch, dass die von den deutschen Eurobetriebsräten praktizierte Vorgehensweise des stellvertretenden Handelns bei ihren mittelosteuropäischen Kollegen umstritten ist. Was von den deutschen Vertretern als eine eigene Vorleistung im Interesse aller verstanden und propagiert wird, ist in den Augen der Vertreter von Standorten aus Mittel- und Osteuropa auch (eigennützige) Interessenpolitik, die die eigene Handlungsautonomie einzuschränken droht (s. hierzu ausführlich Klemm et al. 2011). Dass die Kollegen aus den MOEStandorten noch nicht so recht mitziehen wollen, ist den deutschen Akteuren natürlich nicht entgangen:

„Also die (gemeint sind Interessenvertreter von Standorten in Frankreich, Italien und Spanien, Anm. d. Verf.) haben erkannt, dass wenn sie im europäischen Betriebsrat sind, dass sie dann nen Vorteil davon haben, weil sie kriegen jetzt Kontakte hin, die sie vorher nicht gehabt haben (...) Das ist das Positive an dieser Geschichte, obwohl, wie gesagt, im Ostbereich das immer noch problematisch ist, da jetzt richtig Fuß zu fassen" (deutsches EBR-Mitglied, Fall 2).

Die im Zitat angesprochene Schwierigkeit, im „Osten“ Fuß zu fassen, wird auf deutscher Seite mithilfe von drei Erklärungsstrategien bzw. Deutungsmustern reflektiert. Erstens wird das Verhalten der
Kollegen aus Mittel- und Osteuropa aus der Rückständigkeit der dortigen Vertretungssysteme erklärt:

„(...) das war so'n kleiner Kulturschock für mich, wie wir das erste Mal in (MOELand) waren und dann auch mit Kollegen diskutiert haben und wie wir da auch nur über einige Standards diskutiert haben, was da eigentlich fehlte: So'n Umkleideraum, ja das ist nicht ganz so wichtig, denn wir sind hier dem Herr X (Firmenname) sehr dankbar, dass er hier nen Werk gebaut hat und wir hier (...) arbeiten dürfen. Also diese Mentalität war da“ (deutsches EBRMitglied, Fall 3).

In Mittelosteuropa wird demnach eine effektive Interessenvertretung durch eine postsozialistische, rückständige Mentalität behindert. Das zweite Deutungsmuster kreist um die Frage der Legitimität der betrieblichen Interessenvertreter in Mittelosteuropa:

„Wir haben, wenn wir nach Tschechien fahren, (...) immer ein komisches Gefühl, ob das nun wirklich ne Arbeitnehmervertretung richtig ist" (deutsches EBR-Mitglied, Fall 3).

Aus westlicher Sicht sind die Strukturen betrieblicher Interessenvertretung in Mittelosteuropa undurchsichtig. Anders als im nationalen Handlungsraum ist es für viele Interessenvertreter aus Deutschland schwer, die Zugehörigkeit der mittelosteuropäischen Kollegen zur Arbeitnehmerseite treffsicher einzuschätzen. In der kollektiven Deutung wird das Unvertrautheitsproblem zu einer Eigenschaft der Verhältnisse „im Osten“. Eine dritte Interpretation bezieht sich darauf, missverstanden zu werden:

„Wie gehen wir denn insgesamt miteinander um? Lassen wir uns gegenseitig ausspielen oder versuchen wir, der Geschäftsführung einfach klar zu machen (...), sie kriegen von allen dieselbe Antwort, nämlich wir sind nicht mehr bereit auf irgendwelche Kostensenkungsprogramme einzugehen. Aber es ist halt bei der Diskussion geblieben, weil wir es immer noch nicht geschafft haben, von der einzelbetrieblichen Denkweise wegzukommen in ne gesamte Denkweise, weil halt auch da wieder die Problematik auftritt, aha der deutsche Gewerkschaftsvertreter will uns sagen, was wir zu tun und zu lassen haben" (deutsches EBR-Mitglied, Fall 3).

Diese dritte Form der Reflexion von Kooperationsproblemen verweist im Grunde auf den „Ruf“, den „die Deut- 
schen" im Ausland haben: Ideen aus Deutschland werden als deutsche Ideen zurückgewiesen. In dieser Deutung sind nicht die Absichten das Problem, sondern ein Missverständnis der Absichten, das aus der deutschen Vergangenheit resultiere.

Das Befremden der Kollegen aus Mittel- und Osteuropa ist in der deutschen Perspektive nicht auf das Handeln der deutschen betrieblichen Arbeitnehmervertreter bezogen, sondern eine Folge der mittelosteuropäischen Verhältnisse. Aus der Sicht der betrieblichen Interessenvertreter aus Mittel- und Osteuropa sind es jedoch nicht so sehr die oft widrigen Bedingungen der betrieblichen Arbeitnehmervertretung, die sie dazu veranlassen, skeptisch gegenüber den Einbindungsstrategien der deutschen Betriebsräte zu bleiben. Sie verwehren sich vor allem dagegen, dass das Vertretungshandeln an ihren Standorten und in ihren Ländern sowie des gesamten unternehmensweiten Handlungsraumes der Bewertungshoheit der deutschen Kollegen unterworfen wird, obwohl diese kaum Einsicht in die konkreten Handlungsbedingungen an den mittelosteuropäischen Standorten haben. Eine tschechische Betriebsgewerkschafterin bemerkt:

„Aber trotz alldem tauschen wir uns dort aus, auch wenn sie oft nicht ganz verstehen, wie es bei uns funktioniert (...) Das Umfeld ist bei ihnen völlig anders als bei uns, wir müssen uns wirklich alles erst aufbauen, das mussten sie vielleicht auch, ich weiß nicht, irgendwann nach 1945“ (tschechische Betriebsgewerkschafterin mit internationalen Kontakten, Fall 1).

Dabei sind es die Strategien und die Praktiken des „typischen“ deutschen Betriebsrats bei der Überzeugungsarbeit selbst, die den Verdacht nähren, strategisch vereinnahmt zu werden. Spezifische Praktiken deutscher Arbeitnehmervertreter nämlich, die von allen Akteuren im deutschen System der betrieblichen Interessenvertretung als legitim bewertet werden, die deshalb vertrauensbildend wirken und mit denen sie im nationalen Rahmen erfolgreich waren und auch noch sind, haben in den Augen der Betriebsräte in Mittel- und Osteuropa eine ganz andere Bedeutung: In Deutschland übliche ,gentlemen agreements" und deren Bedingung, eine recht enge Vertrautheit zwischen Managementvertretern und Betriebsratsvertretern, stehen unter dem Verdacht der Kumpanei; die großen Reden der Deutschen gegen das Management, etwa auf Tagungen des
Eurobetriebsrats, erregen Skepsis, die Diskrepanz zwischen der „verbalen Wucht“, mit der deutsche Betriebsräte z.B. auf Betriebsversammlungen dem Management gegenübertreten, und der Vertrautheit zwischen lokalem Standortmanagement und Eurobetriebsrats-Mitgliedern ist zumindest befremdlich.

„(...) ich sagte, Herrgott, was für...was für ein Kunde kann das sein, so wichtig, der Personalchef springt da rum, auch der Produktionschef, der Direktor, wer kann das bloß sein, was für ein hoher Rang, und das war der (Euro-)Betriebsrat" (tschechischer EBR, Fall 1).

Die Betonung der persönlichen Bekanntschaft mit und das informelle Gespräch über Spitzenmanager, in Deutschland eine Selbstverständlichkeit, zumindest dort, wo die Arbeitnehmervertretung stark ist, läuft der Alltagserfahrung mit dem eigenen Standortmanagement diametral entgegen. In Deutschland ist die persönliche Nähe der Betriebsratsspitzen zu Top-Managern Ausdruck der eigenen Stärke und Zeichen der dem Management aufgezwungenen Anerkennung der Position der Arbeitnehmervertretung. In Mittelosteuropa ist ein solches Verhältnis kaum vorstellbar. Die Erfahrung der betrieblichen Interessenvertreter ist vielmehr, dass man dem Management prinzipiell nicht vertrauen kann und nur rechtlich einklagbare Abmachungen einen Sinn haben. Ein ungarischer Betriebsrat schildert seine Erfahrung so:

„(...) ich habe den Kollektivvertrag hervorgenommen (...) und ich habe den verehrten deutschen Kollegen gefragt (schlägt auf den Tisch), ob er weiß, was dieses Dokument ist. Er sagte: nein. Nun dann, es ist der Kollektivvertrag. Er ist hier das Gesetz. Und wenn ich aufblättere, und ich habe ohne Großtuerei aufgeschlagen, ich habe ihn angeschaut, und habe gesagt, meine Unterschrift und die Unterschrift von XX steht darin. Ihre nicht! Also ich habe kein Rechtsverhältnis mit Ihnen. Sie haben keine Berechtigung, dieses Gesetz zu revidieren. Falls Sie Fragen haben, Management, dann können wir das besprechen. Also das ist kein Ghana, keine Elfenbeinküste (...) (ungarischer BR, Fall 1).

Die deutschen Interessenvertreter versuchen, dem ihnen entgegengebrachten Misstrauen konsequent mit den schon genannten Mitteln zu begegnen: der Demonstration erfolgreicher Intervention beim Zentralmanagement, der Dokumentation eigener Macht durch Betonung der persönlichen Verbindungen ins zentrale Management etc. Die Kooperationsbereitschaft der mittelosteuropäischen Vertreter sowie deren Einlenken auf ein Vertretungsmodell nach Vorstellung der deutschen Arbeitnehmervertreter werden zunehmend offensiv eingefordert. Wo dieses Einlenken sich nicht abzeichnet, wird die Möglichkeit, selbst aktiv in den Standortwettbewerb - auch gegen die ostmitteleuropäischen Standorte - einzutreten, in Aussicht gestellt oder faktisch praktiziert. Aus der Gegenperspektive verstärkt sich so einerseits die Sicht des „Einverleibtwerdens“ als eines mehr oder minder durchschaubaren Manövers und andererseits der Verdacht, dass ein konkretes Interesse, die Handlungsbedingungen in Mittelosteuropa so kennenzulernen, wie sie sich aus ihrer Perspektive darstellen, gar nicht vorhanden ist. Da dies wiederum Voraussetzung dafür wäre, die dortigen Interessen zu berücksichtigen, erscheint es naheliegend, den Vereinnahmungstendenzen entgegenzutreten bzw. selbst für die Vertretung der eigenen Interessen Sorge zu tragen.

„(...) mein Gefühl dabei war immer, dass sie uns zwar vor Jahren sozusagen respektiert haben, aber wollten nicht mit uns viel kommunizieren, aber jetzt, nachdem sich das Kapital immer mehr Richtung Osten verschiebt, da empfinden sie uns logischerweise als riesige Bedrohung, und heute legen sie eigentlich großen Wert auf Kommunikation, jetzt sind sie sehr an Informationen interessiert, die wir ihnen geben können. Und wir empfinden das selbstverständlich so, dass wir früher ihnen nicht gut genug waren, um mit uns zu kommunizieren, und jetzt merken wir selbstverständlich (...), sodass wir einige kleine Geheimnisse in dieser Beziehung hüten, weil sie es früher genauso gemacht haben, wir hätten früher Hilfe gebrauchen können und wir haben sie wirklich nicht gespürt" (tschechischer Betriebsgewerkschafter, Fall 1).

Während also die deutschen Vertreter Handlungsmuster in der internationalen Zusammenarbeit anwenden, die sie auch in Deutschland mit Erfolg anwenden, bewerten andere, die mit diesem Modell nicht vertraut sind, diese Handlungsmuster nicht in erster Linie als vertrauensbildende Formen anerkannten und richtigen Interessenvertretungshandelns, sondern als undurchsichtige Strategien der Vereinnah- 
mung - das Gleiche ließe sich umgekehrt genauso rekonstruieren. Da internationale Treffen in der Regel kurz und sporadisch stattfinden und dabei erhebliche sprachliche Verständigungshürden zu überwinden sind, existieren kaum gemeinsame soziale Settings, in denen die genannten unterschiedlichen Bewertungen zur Sprache gebracht werden könnten. Unterschiedliche Sichtweisen werden so nicht zugänglich gemacht, sondern bleiben selbst bei faceto-face Kontakten im Rahmen von Eurobetriebsratstreffen in vielen Fällen latent. Im jeweiligen nationalen Kontext verstärkt sich der Eindruck der Rückständigkeit einerseits oder der Vereinnahmung andererseits. Im Ergebnis wird eine im nationalen Rahmen gut etablierte Strategie des Vertretungshandelns im internationalen Rahmen problematisch.

\section{Zusammenfassung und Diskussion}

Das selbstverständliche „Einverständnishandeln" (siehe in Anlehnung an Max Weber: Schmidt 1986) zwischen Management und betrieblicher Interessenvertretung hat in westdeutschen Industrieunternehmen durch die erweiterten Optionen des Managements, insbesondere zur Verlagerung von Produktionslinien, eine deutliche Eintrübung erfahren (s. u.a. Heidling et al. 2004). Die zunehmende internationale Orientierung auf Seiten der deutschen Betriebsräte, die sich darin zeigt, die übernationale europäische Ebene für die betriebliche Interessenvertretung zu nutzen, folgt in diesem Sinne gewissermaßen der Internationalisierung des Managements und zielt nicht zuletzt darauf, die erweiterten Handlungsoptionen des Management wieder ein Stück weit einzugrenzen. ${ }^{6}$ Das Vorgehen der deutschen Betriebsräte ,extrapoliert" dabei, wie gezeigt, die Kultur des eigenen Interessenvertretungsmodells auf die europäische Ebene: Man versucht, das Vertretungsmandat auf die ,ausländi- schen" Standorte zu erweitern und so die eigene Position gegenüber dem in Deutschland beheimateten Zentral-Management zu stärken.

Diese völlig legitime Strategie basiert auf der Annahme, man sitze am Ende doch im gleichen Boot und sei in gleicher Weise von den Unternehmenspolitiken des Managements betroffen, weshalb die Ausweitung des Vertretungsmandats doch allen zugute komme. Doch diese - im Rahmen des gestuften Modells deutscher betrieblicher Interessenvertretung naheliegende - Inanspruchnahme legitimer Machtkonzentration wird so nicht von den betroffenen Kollegen in Mittelosteuropa mitgetragen, sie fühlen sich vereinnahmt.

Dass die Kollegen aus Mittel- und Osteuropa der Vorgehensweise der westdeutschen Interessenvertreter skeptisch gegenüberstehen, erklärt sich aus der westlichen Perspektive jedoch weniger aus unterschiedlichen Ausgangslagen, sondern wird mit sozialisatorischen Defiziten, Vorbehalten gegenüber Deutschen und/oder illegitimem Interessenhandeln in Verbindung gebracht. Einerseits gelten die Standorte in Mittel- und Osteuropa als Profiteure, die willentlich westliche Standards unterlaufen (und so dem Westen Produktion „wegnehmen"), andererseits gelten sie als zurückgeblieben. Dies wird den mittelund osteuropäischen Kollegen manifest oder latent auch vermittelt. Der Vorwurf, der Osten habe bisher nur (auf Kosten der Weststandorte) von der EU-Erweiterung profitiert, muss aus Sicht der mittel- und osteuropäischen Arbeitnehmervertreter auf Unverständnis stoßen, wenn man die Verwerfungen nach dem Ende des planbürokratischen Wirtschaftssystems betrachtet. Aus mittelosteuropäischer Perspektive handelt es sich nicht um ein "Profitieren,“ bei dem man anderen etwas weggenommen hat, sondern um einen Kampf unter schwierigen Bedingungen in einem System Industrieller Beziehungen, das sie selber mit aufgebaut haben. Sie sehen sich selbst als Handelnde unter widrigen Bedingungen, die trotz Transformation Arbeitnehmerrechte auch gegen eine neoliberale Aus- verkaufstrategie der eigenen Regierungen bewahrt und ausgebaut haben, die zudem nicht verantwortlich für die Managemententscheidungen der westlichen Konzernzentralen gemacht werden können. Die Sicht der deutschen Betriebsräte auf die Zustände „im Osten“ - Zustände wie im Frühkapitalismus, defizitäre betriebliche Interessenvertretung usw. - sehen von deren Handlungsbedingungen und -erfolgen fast vollständig ab. Hinzu kommt, dass das im nationalen Rahmen als Grundlage vertrauensvoller Zusammenarbeit völlig selbstverständliche Auftreten deutscher Betriebsräte im internationalen Kontext oftmals den gegenteiligen Effekt erzeugt: Das selbstbewusste Auftreten läuft Gefahr, als Arroganz wahrgenommen zu werden, und der Umgang mit dem Management als Kumpanei. Umgekehrt wird die zurückhaltendere Variante des Auftretens ostmitteleuropäischer Vertreter als fehlendes Selbstbewusstsein registriert. Das Agieren deutscher EBR-Mitglieder droht so - trotz möglicher Erfolge und Vorteile auch für die nichtdeutschen Standorte - ins Leere zu laufen.

Abschließend lässt sich festhalten, dass die Durch- und Umsetzbarkeit von Handlungsstrategien im europäischen Rahmen nicht nur an Interessenunterschieden, sondern auch an kulturellen Faktoren scheitern kann. Zumindest nach unseren Daten sind viele Belegschaftsvertreter in Mittelosteuropa nicht bereit, eine westliche Definition ihrer nationalen und lokalen Handlungssituationen zu akzeptieren, die ihnen eine inferiore Position zuweist. Strategien und Kooperationen werden darum bemüht sein müssen, unterschiedliche Situationsdefinitionen zuzulassen und sich nicht davon abhängig zu machen, dass zunächst die spezifisch deutsche Perspektive auf den europäischen Interessenvertretungsraum von allen Akteuren akzeptiert wird.

\footnotetext{
6 Wo ein solcher Bedarf nicht vorhanden ist, fehlt häufig auch das Interesse von Seiten deutscher Betriebsräte an der Gründung von Eurobetriebsräten (siehe Whitall et al. 2009).
} 
Dehnen, V. (2010): Die Einbindung von mittel- und osteuropäischen Standorten in die Arbeit von Europäischen Betriebsräten - Interessensausgleich zwischen EBR-Mitgliedern am Beispiel General Motors, in: Industrielle Beziehungen 17 (2), S. 147-169

Eberwein, W./Tholen, J./Schuster, J. (2000): Die Europäisierung der Arbeitsbeziehungen als politisch-sozialer Prozeß, München/Mering Hauser-Ditz, A./Hertwig, M./Pries, L./Rampeltshammer, L. (2010): Transnationale Mitbestimmung? Zur Praxis Europäischer Betriebsräte in der Automobilindustrie, Frankfurt/New York

Heidling, E./Deiß, M./Meil, P./Schmierl, K. (2004): Restrukturierung nationaler Interessenvertretung, in: Beck, U./Lau, C. (Hrsg.): Entgrenzung und Entscheidung: Was ist neu an der Theorie reflexiver Modernisierung?, Frankfurt/M., S. 360-383

Hürtgen, S. (2008): Transnationales Co-Management. Betriebliche Politik in der globalen Konkurrenz, Münster

Klemm, M./Kraetsch, C./Weyand, J. (2011): Chancen kulturübergreifender Solidarität in global agierenden Unternehmen: Kommunikation, Macht und Interessen in der transnationalen Arbeitnehmerkooperation, Abschlussbericht (Publikation in Vorbereitung)

Klemm, M./Weyand, J. (2009): Communication and solidarity in crosscultural employee relations, in: Hertwig, M./Pries, L./Rampeltshammer, L. (Hrsg.): European Works Councils in complementary perspectives. New approaches to the study of European interest regulation, Brussels, S. $261-279$

Kotthoff, H. (1998): Mitbestimmung in Zeiten interessenpolitischer Rückschritte. Betriebsräte zwischen Beteiligungsofferten und "gnadenlosem Kostensenkungsdiktat" ${ }^{\prime}$, in: Industrielle Beziehungen 5 (1), S. $76-100$

Kotthoff, H. (2005): EU-Osterweiterung: die aktuelle Herausforderung für den Europäischen Betriebsrat, Düsseldorf

Kotthoff, H. (2006): Lehrjahre des Europäischen Betriebsrats. Zehn Jahre transnationale Arbeitnehmervertretung, Berlin Lecher, W./Nagel, B./Platzer, H.-W. (1998): Die Konstituierung Europäischer Betriebsräte - Vom Informationsforum zum Akteur?, BadenBaden
Lecher, W./Platzer, H.-W./Rüb, S./Weiner, K.-P. (2001): Verhandelte Europäisierung. Die Einrichtung Europäischer Betriebsräte - Zwischen gesetzlichem Rahmen und sozialer Dynamik, Baden-Baden Lücking, S./ Trinczek, R./ Whittall, M. (2008): Europäische Betriebsräte: Was lehrt der Fall für die Revision der EU-Richtlinie?, in: WSIMitteilungen 61 (5), S. 246-253

Müller, T./Hoffmann, A. (2001): EWC Research: A Review of the Literature, Warwick Papers in Industrial Relations (65) (letzter Zugriff März 2009: http://www2.warwick.ac.uk/fac/soc/wbs/research/irru/ wpir/wpir65.pdf)

Müller, T./Platzer, H.-W./Rüb, S. (2004): Globale Arbeitsbeziehungen in globalen Konzernen?, Wiesbaden

Schmidt, G. (1986): Einverständnishandeln - ein Konzept zur "handlungsnahen“ Untersuchung betrieblicher Entscheidungsprozesse, in: Seltz, R./Mill, U./Hildebrandt, E. (Hrsg.): Organisation als soziales System. Kontrolle und Kommunikationstechnologie in Arbeitsorganisationen, Berlin, S. 57-70

Srubar, I. (1998): Ethnicity and Social Space, in: Balla, B./Sterbling, A. (Hrsg.): Ethnicity, Nation, and Culture. Central and East European Perspectives, Hamburg, S. 47-64

Stirling, J./Tully, B. (2004): Power, Process, and Practice: Communication in European Works Councils, in: European Journal of Industrial Relations 10 (1), S. 73-89

Swidler, A. (1986): Culture in Action: Symbols and Strategies, in: American Sociological Review 51 (2), S. 273-286

Voss, E. (2006): The experience of European Works Councils in new EU Member States, hrsg. von der European Foundation for the Improvement of Living and Working Conditions, Dublin

Whittall, M./Lücking, S./Trinczek, R. (2008): Understanding the European Works Councils deficit in German multinationals, in: transfer 14 ( 3), S. 453-467

Whittall, M./Lücking, S./Trinczek, R. (2009): The frontiers within: why employee representatives fail to set up European works councils, in: Industrial Relations Journal 40 (6), S. 546-562 